\title{
Correction to: Weld Zone Characterization of Dissimilar SS UNS S43000/UNS S32304 Joined through Laser Beam Welding: EBSD Study, Phase Evolutions, and Fractography
}

Hossein Mostaan, Morteza Shamanian, Reza Moradi, Fardin Nematzadeh, Ali Sonboli, and Jerzy A. Szpunar

\section{Correction to:}

JMEPEG (2020) 29:7744-7754

https://doi.org/10.1007/s11665-020-05239-1

Readers should note that the name of the second author of this article was misspelled (as Morteza Shmanian) in the article as published.
The correct spelling of the author's name is Morteza Shamanian.

Publisher's Note Springer Nature remains neutral with regard to jurisdictional claims in published maps and institutional affiliations.

The original article can be found online at https://doi.org/10.1007/ s11665-020-05239-1.

Hossein Mostaan, Reza Moradi, Fardin Nematzadeh, and Ali Sonboli, Faculty of Engineering, Department of Materials and Metallurgical Engineering, Arak University, Arak 38156-8-8349, Iran; Morteza Shamanian, Department of Materials Engineering, Isfahan University of Technology, Isfahan 84156-83111, Iran; Jerzy A. Szpunar, Department of Mechanical Engineering, University of Saskatchewan, Saskatoon, SK S7N 5A9, Canada. Contact e-mail: H-mostaan@araku.ac.ir. 